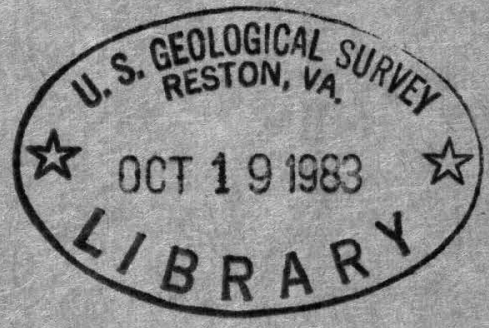


UNITED STATES DEPARTMENT OF THE INTERIOR

GEOL OGICAL SURVEY

MOL AR VOLUMES AND DENSITIES OF MINERALS *

By

Richard A. Robie and Philip M. Bethke

July 1962

Report TEI-822

This report is preliminary and has not been edited for conformity with Geological Survey format.

* This work was supported in part by the Division of Reactor Development, U. S. Atomic Energy Commission. 

No. of copies

Division of Reactor Development (W. G. Belter) . ....... Division of Raw Materials (R. D. Nininger) . . . . . . . Division of Peaceful Nuclear Explosives (R. Hamburger) . . . . Division of Research (D. R. Milier)............ Hanford operations office (C. I. Robinson) . . . . . . . . Grand Junction operations office ............. Idaho operations office (John Horan) . . . . . . . . . Oak Ridge Operations office (H. M. Roth) ........... Savannah River operations office (Karl Herde). ....... Office of Technical Information Extension, oak Ridge ..... Nevada Operations Office (James E. Reeves) ........... Office of Operations Analysis and Plaming, Washington .... U. S. Naval Radiological Defense Lab., San Francisco ..... Health Physics Division, Oak Ridge National Laboratory

(E. G. Struxness and F. I. Parker) ....... Chemistry Division, Argonne National Lab.(W. M. Manning. . . . Chemical Tech. Div., Oak Ridge National Lab. (W. E. Clark) .. U. So Bureau of Mines, Bartlesville, Oklahoma. . . ...... Ios Alamos Scientific Laboratory (J。 H. Hall) . . . . . . . Ios Alamos Scientific Laboratory (C. W. Christenson) ...... Earth Sciences Division, NAS-NRC (Iim Hoover) . . . . . . University of Texas, Austin (E. F. Gloyna) . . . . . . . . General Electric Company, Richland, Washington (E. R. Irish) . University of California (W. J. Kaufman) . . . . . . . . E. I. DuPont deNemours and Company (C. M. Patterson) ...... Lawrence Radiation Iaboratory, Technical Information

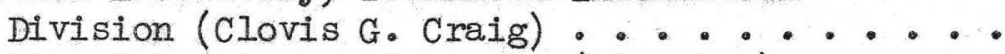
Lawrence Radiation Laboratory, Livermore (Director)......

U. S. Geological Survey: Geologic Division. ... . . . . . . . . . . . . . Water Resources Division .................. Total 
1 


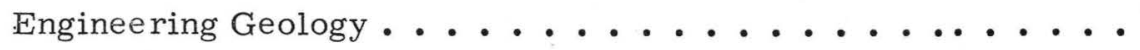

Geochemical Exploration and Minor Elements .......

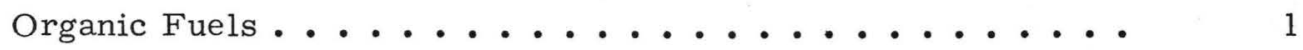

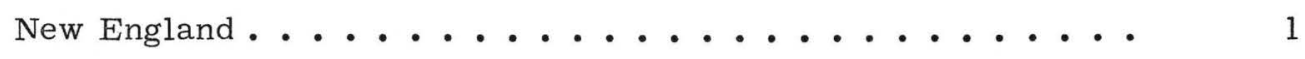

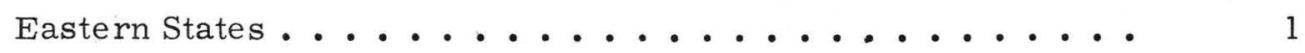

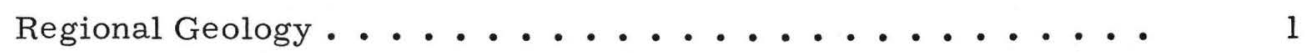

Southern Rocky Mountains ................ 2

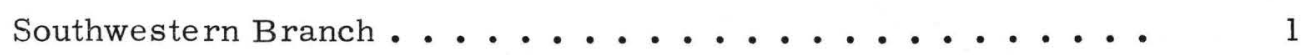

Pacific Coast States ........................... 1

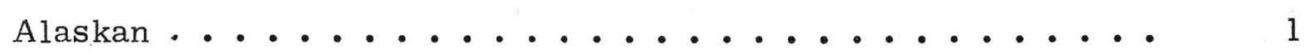

Regional Geophysics........................... 4

Experimental Geochemistry and Mineralogy .......... 1

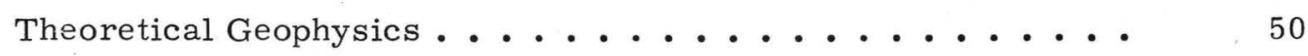

Isotope Geology ..................... 1

Field Geochemistry and Petrology ............. 2

Analytical Laboratories ................. 1

Foreign Geology ...................... 1

Military Geology .......................... 1

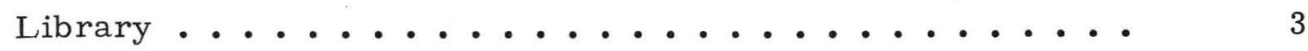

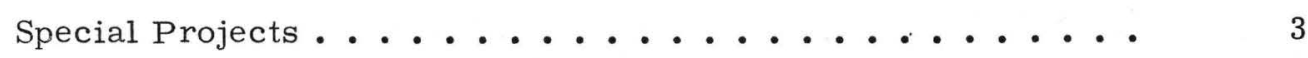

Geologic Division............................. $\frac{17}{97}$ 



\section{MOLAR VOLUMES AND DENSITIES OF MINERALS}

By Richard A. Robie and Philip M. Bethke

These tables present critically chosen "best values" for the density and molar volume of selected mineral compounds. No attempt was made to be all-inclusive; rather we have tried to present data for chemically and physically well-defined phases for which the molar volume and/or density was known to the order of 0.2 percent.

Data are included for several materials not known as minerals but for which the data are necessary for the calculation of partial molar volumes of intermediate members of a solid solution. For similar reasons we have also included a few values of hypothetical phases (e.g. FeS wurtzite and sphalerite structures, and CdS rock salt structure) based on the extrapolation of measured cell dimensions from incomplete solid solutions. These theoretical phases are so indicated in the tables. The majority of the data included are for pure synthetic phases for which unit cell parameters or densities have been determined with an accuracy of 0.1 percent or better. In some instances, where the mineral deviates from the stoichiometric composition, either by substitutional or omission solid solution, one or more values are given for materials of known composition as noted in the Formula column.

For several substances, measured densities having equal or greater accuracy than the $\mathrm{X}$-ray data are available. For these compounds the molar volume was calculated from (1), and the uncertainty listed with the density.

Molar volumes were calculated from measured densities by the relation:

$$
\mathrm{V}=\frac{\mathrm{M}}{\mathrm{p}}
$$


where $\mathrm{M}$ is the formula weight in grams and $\rho$ is the density in grams $^{-3}$, or from the unit cell dimensions,

$$
\mathrm{V}=\frac{\text { (unit cell volume in } \mathrm{cm}^{3} \text { ) (Avogadro's number) }}{\text { (number of formulas)/(unit cell) }}
$$

Formulas for the volumies of various shaped unit cells are given in Barrett (1952).

Substances of trigonal symmetry are denoted by the symbol hex- $\mathrm{R}$ to distinguish them from materials of truly hexagonal symmetry. The cell volume and number of formula weights listed for these compounds are however given for the larger hexagonal cell.

Substances denoted by an asterisk indicate the data were obtained from natural mineral specimens whose composition may have deviated slightly from the listed composition. Densities given for these minerals were calculated using the formula weight for the stoichiometric phase.

The formula weights are based on the International Atomic Weights for 1957 (Wichers, 1958). Avogadro's number used for these tables is (6. 02322士.00016) $\times 10^{23}$ particles mole ${ }^{-1}$ which is the physical value of Cohen, Crowe, and DuMiond (1957) converted to the chemical scale, using the conversion factor $1.00027 \pm .000005$ of these authors. Temperatures for which the volumes and density apply are given in the second column from the right. Molar volumes for gases refer to the real gas at one atmosphere pressure. The letter $\underline{\mathbf{r}}$ indicates that the measurements were made at an unspecified room temperature. This may be taken as $25^{\circ} \pm 5^{\circ} \mathrm{C}$. Due to a lack of adequate thermal expansion data, no attempt was made to reduce all the data to the common reference temperature, $25^{\circ} \mathrm{C}$. The uncertainties given for the molar volumes include an estimate of the precision, the reproducibility between different investigators and in some cases slight deviations from stoichiometry. Although the uncertainties are listed only 
for the molar volumes, it must be understood that an equal percentage uncertainty is associated with the cell volume and the density.

We wish to acknowledge the considerable help rendered in the preparation of these tables by our colleagues at the U. S. Geological Survey, particularly Mrs. Martha S. Toulmin who aided greatly in compiling and checking the unit cell parameters of the sulfides and related minerals and Jerry L. Edwards who checked the majority of the non-sulfide data and prepared the bibliography.

References

Barrett, Structure of Metals, McGraw-Hill Book Co., New York, 1952 . Wichers, J. Am. Chem. Soc. 80, 4121, 1958.

Cohen, Crowe, and DuMond, Fundamental Constants of Physics, Interscience Pub., New York, 1957. 
ELEMENTS

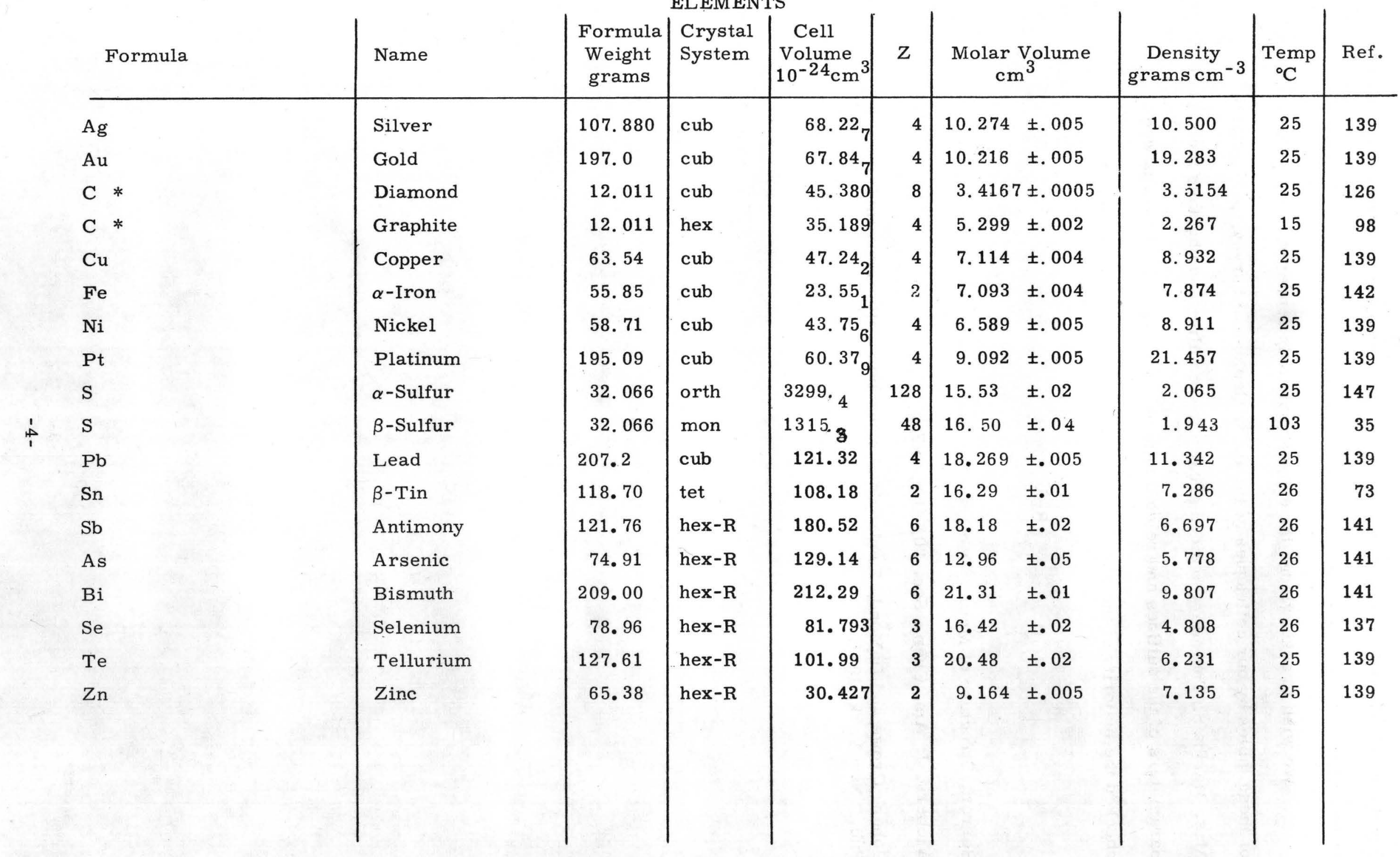


SULFIDES, TELLURIDES, SELENIDES, AND ARSENIDES

\begin{tabular}{|c|c|c|c|c|c|c|c|c|c|}
\hline Formula & 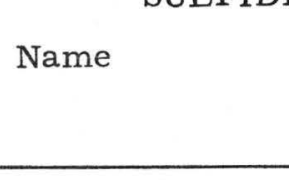 & $\begin{array}{c}\text { Formula } \\
\text { Weight } \\
\text { grams }\end{array}$ & $\begin{array}{l}\text { Crystal } \\
\text { System }\end{array}$ & $\mid \begin{array}{l}\text { Cell } \\
\text { Volume } \\
10^{-24} \mathrm{~cm}^{3} \\
\end{array}$ & Z & $\begin{array}{c}\text { Molar Volume } \\
\mathrm{cm}^{3}\end{array}$ & $\begin{array}{c}\text { Density } \\
\text { grams cm }^{-3}\end{array}$ & $\begin{array}{c}\text { Temp } \\
{ }^{\circ} \mathrm{C}\end{array}$ & Ref. \\
\hline$\beta-\mathrm{Ni}_{3} \mathrm{~Pb}_{2} \mathrm{~S}_{2}$ * & Shandite & 651.682 & hex & $367 .{ }_{6}$ & 3 & $73.8 \quad \pm .1$ & 8.827 & $r$ & 71,107 \\
\hline $\mathrm{Au}_{2} \mathrm{Bi}$ & Maldonite & 603.00 & cub & $503 .{ }_{8}$ & 8 & $37.94 \pm .01$ & 15.892 & $\mathbf{r}$ & 75 \\
\hline $\mathrm{Ag}_{2} \mathrm{~S} \mathrm{I}$ & Hi-Argentite & 247.826 & cub & 246.37 & 4 & $37.1 \pm .2$ & 6.680 & 600 & 32 \\
\hline $\mathrm{Ag}_{2} \mathrm{~S} I I$ & Argentite & 247.826 & cub & 115.50 & 2 & $34.8 \pm .1$ & 7.125 & 189 & 32 \\
\hline $\mathrm{Ag}_{2} \mathrm{~S}$ III & Acanthite & 247.826 & orth & 227.08 & 4 & $34.2 \pm .2$ & 7.254 & $r$ & 32,148 \\
\hline $\mathrm{Ag}_{2} \mathrm{Se}$ & Hi-Naumannite & 294.72 & cub & 124.48 & 2 & $37.5 \pm .2$ & 7.862 & 170 & 113 \\
\hline $\mathrm{Ag}_{2} \mathrm{Te} \mathrm{I}$ & & 343.37 & cub & 158.6 & 2 & $44.6 \pm .2$ & 7. 702 & 825 & 113 \\
\hline $\mathrm{Ag}_{2} \mathrm{Te} I \mathrm{II}$ & & 343.37 & cub & 285.5 & & $43.0 \quad \pm .1$ & 7.986 & 250 & 113 \\
\hline $\mathrm{Ag}_{2} \mathrm{Te}$ III $*$ & Hessite & 343.37 & mon & 271.4 & 4 & $40.86 \pm .20$ & 8.405 & $r$ & 49 \\
\hline $\mathrm{Cu}_{2} \mathrm{~S} \mathrm{I} *$ & Hi-Digenite & 159.146 & cub & 187.6 & 4 & $28.26 \pm .10$ & 5.632 & 465 & 31, \\
\hline $\mathrm{Cu}_{2} \mathrm{~S}$ II * & Hi-Chalcocite & 159.146 & hex & 97.16 & & $27.51 \pm .06$ & 5.786 & 152 & 31, \\
\hline $\mathrm{Cu}_{2} \mathrm{~S}$ III $*$ & Chalcocite & 159.146 & $\begin{array}{l}\text { orth } \\
\text { pseudo- }\end{array}$ & 4379.5 & 96 & $27.48 \pm .01$ & 5.792 & $r$ & 31, \\
\hline $\mathrm{Cu}_{1.79^{\mathrm{S}} \quad(\mathrm{Cu} \text { rich side })}$ & Digenite & 145.803 & cubic & $172.7_{6}$ & 4 & $26.01_{5} \pm .01$ & 5.605 & 25 & 116 \\
\hline $\mathrm{Cu}_{1.77^{-}} \mathrm{S} \quad$ (S rich side) & Digenite & 144.532 & $\begin{array}{l}\text { pseudo- } \\
\text { cubic }\end{array}$ & 171.34 & 4 & $25.80 \pm .01$ & 5.602 & 25 & 31,116 \\
\hline $\mathrm{Cu}_{2} \mathrm{Se}$ & Berzelianite & 206.04 & eub & 200.2 & 4 & $30.15 \pm .15$ & 6.835 & 170 & 113 \\
\hline $\mathrm{Ag}_{1.55^{\mathrm{Cu}}} \mathrm{u}_{0.45^{\mathrm{S} \mathrm{I}}}$ & & 227.873 & cub & $228.1_{0}$ & 4 & $34.35 \pm .10$ & 6.634 & 300 & 32 \\
\hline $\mathrm{Ag}_{1.55} \mathrm{Cu}_{0.45} \mathrm{~S} \mathrm{II}$ & & 227.873 & cub & $123.3_{3}$ & 2 & $33.83 \pm .10$ & 6.736 & 116 & 32 \\
\hline $\mathrm{Ag}_{1.55} \mathrm{Cu}_{0.45} \mathrm{~S} \mathrm{III}$ & Jalpaite & 227.873 & tet & 884.30 & 16 & $33.29 \pm .03$ & 6.845 & $\mathrm{r}$ & 32 \\
\hline $\mathrm{Ag}_{0.93} \mathrm{Cu}_{1.07} \mathrm{~S} \mathrm{I}$ & & 200.382 & cub & 211.8 & 4 & $31.90 \pm .10$ & 6.282 & 196 & 32 \\
\hline $\mathrm{Ag}_{0.93} \mathrm{Cu}_{1.07} \mathrm{~S} \mathrm{II}$ & & 200.382 & hex & 105.4 & 2 & $31.73 \pm .10$ & 6.315 & 100 & 32 \\
\hline
\end{tabular}




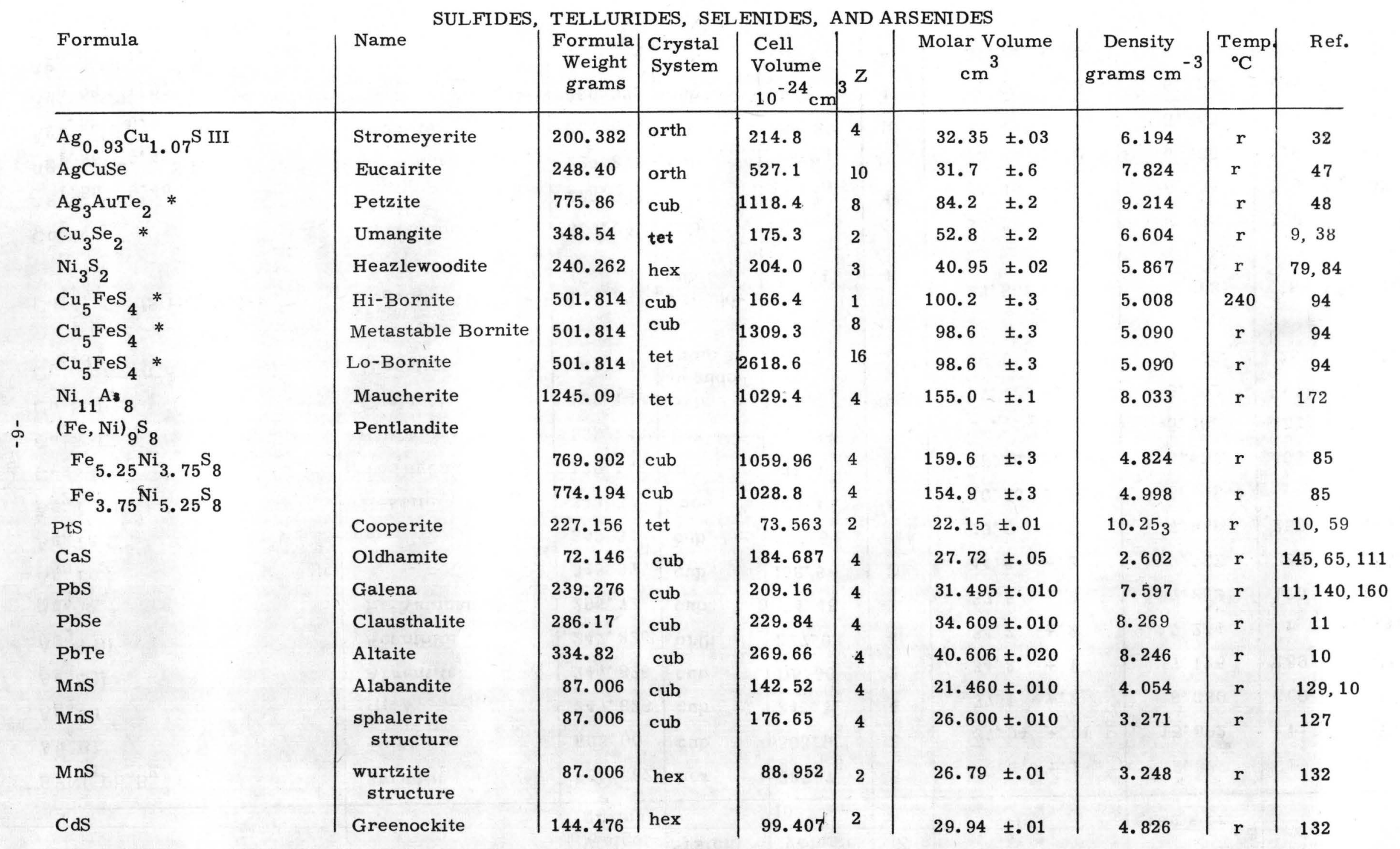


SULFIDES, TELLURIDES, SELENIDES, AND ARSENIDES

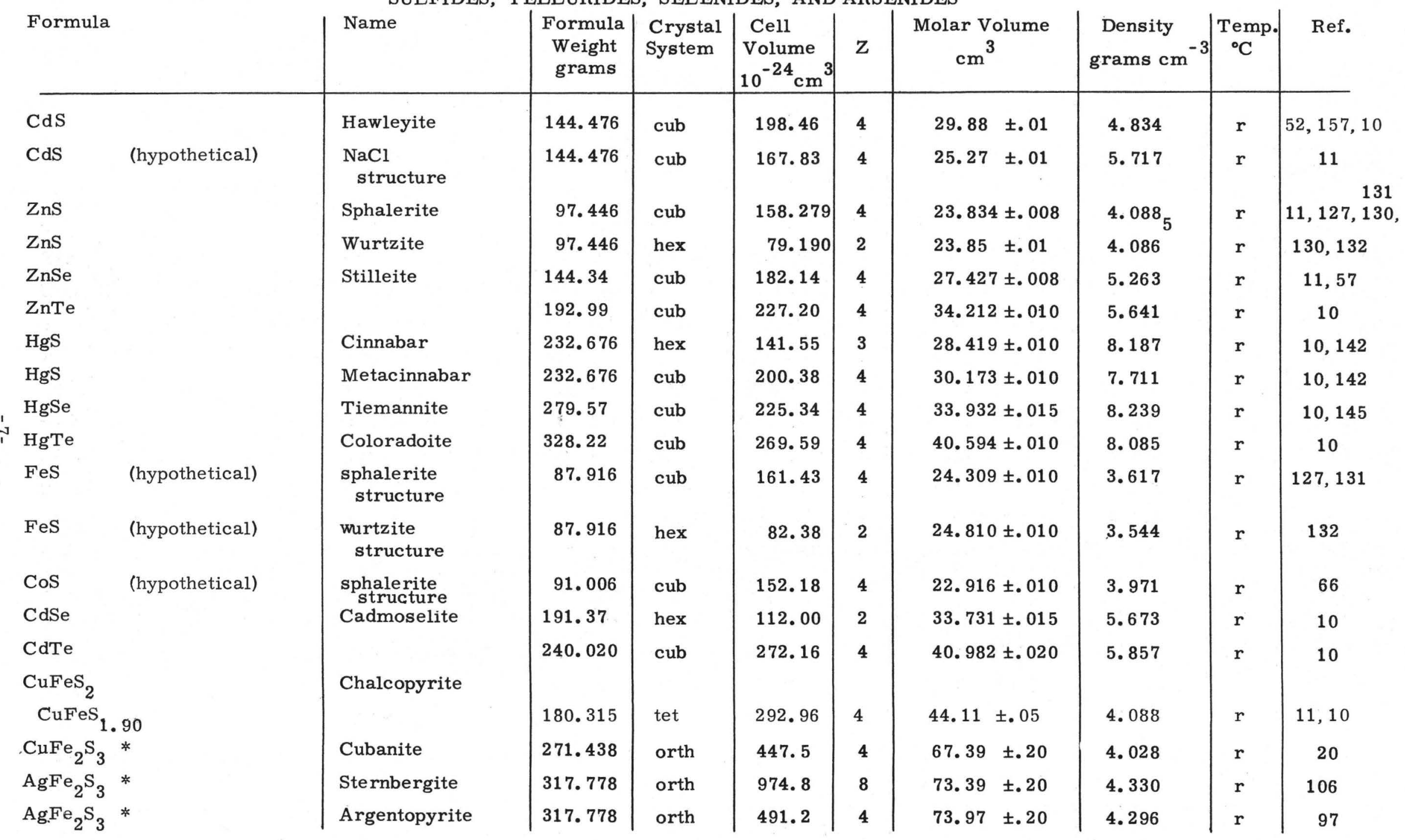


SULFIDES, TELLURIDES, SELENIDES, AND ARSENIDES

\begin{tabular}{|c|c|c|c|c|c|c|c|c|c|}
\hline Formula & $\begin{array}{l}\text { SULFIDES, } \mathrm{T} \\
\text { Name }\end{array}$ & $\begin{array}{l}\text { LLURIDES } \\
\text { Formula } \\
\text { Weight } \\
\text { grams }\end{array}$ & $\begin{array}{l}\text { SELEN } \\
\text { Crystal } \\
\text { System }\end{array}$ & $\begin{array}{l}\text { DES, AND } \\
\text { Cell } \\
\text { Volume } \\
10^{-24} \mathrm{~cm}^{3} \\
\end{array}$ & Z & $\begin{array}{c}\text { Molar Volume } \\
\mathrm{cm}^{3}\end{array}$ & $\begin{array}{c}\text { Density } \\
\text { grams } \mathrm{cm}^{-3}\end{array}$ & Temp & Ref. \\
\hline NiAs & Niccolite & 113.62 & hex & 57.07 & 2 & $17.186 \pm .020$ & 6.611 & $\mathbf{r}$ & 172 \\
\hline $\mathrm{NiSb}$ & Breithauptite & 180.47 & héx & 69.37 & 2 & $20.893 \pm .030$ & 8.639 & $\mathbf{r}$ & 70 \\
\hline CuS & Covellite & 95.606 & hex & 203.48 & 6 & $20.427 \pm .020$ & 4.680 & $\mathbf{r}$ & 142 \\
\hline CuSe & Klockmannite & 142.50 & hex & 3014.8 & 78 & $23.257 \pm .020$ & 6.128 & $\mathbf{r}$ & 37,150 \\
\hline $\mathrm{SnS}$ & Herzenbergite & 150.776 & orth & 192.66 & 4 & $29.010 \pm .020$ & 5.197 & $\mathbf{r}$ & 95 \\
\hline $\mathrm{PbSnS}_{2}$ & Teallite & 390.042 & orth & 199.24 & 2 & $60.002 \pm .040$ & 6.500 & $\mathbf{r}$ & 95 \\
\hline $\mathrm{AsS} *$ & Realgar & 106.976 & mon & 791.6 & 16 & $29.82 \pm .10$ & 3.59 & $\mathbf{r}$ & 1684 \\
\hline NiS & Millerite & 90.776 & hex & 252.41 & 3 & $16.891 \pm .008$ & 5.374 & $\mathbf{r}$ & $10,149,79$, \\
\hline $\mathrm{Sb}_{2} \mathrm{~S}_{3}$ & Stibnite & 339.718 & orth & 487.54 & 4 & $73.414 \pm .05$ & 4.696 & 25 & 143 \\
\hline $\mathrm{Bi}_{2} \mathrm{~S}_{3}$ & Bismuthinite & 514.198 & orth & 501.59 & 4 & $75.529 \pm .07$ & 6.808 & 26 & 142 \\
\hline $\mathrm{Bi}_{2} \mathrm{Te}_{3}$ & Tellurobismuthite & 800.83 & hex & 507.32 & 3 & $101.86 \pm .10$ & 7.862 & $\mathbf{r}$ & 45,161 \\
\hline $\mathrm{As}_{2} \mathrm{~S}_{3} *$ & Orpiment & 246.018 & mon & 468.3 & 4 & $70.52 \pm .20$ & 3.49 & $\mathbf{r}$ & 19 \\
\hline $\mathrm{Co}_{3} \mathrm{~S}_{4}$ & Linnaeite & 305.084 & cub & 830.85 & 8 & . $62.55 \pm .05$ & 4.877 & $\mathbf{r}$ & 87 \\
\hline $\mathrm{Ni}_{3} \mathrm{~S}_{4}$ & Polydymite & 304.394 & cub & 851.97 & 8 & $64.14 \pm .01$ & 4. 746 & $\mathbf{r}$ & 79 \\
\hline $\mathrm{FeNi}_{2} \mathrm{~S}_{4}$ & Violarite & 301.534 & cub & 847.66 & 8 & $63.82 \pm .05$ & 4. 725 & $\mathbf{r}$ & 86 \\
\hline $\mathrm{FeCr}_{2} \mathrm{~S}_{4}$ & Daubreeite & 288.134 & cub & 989.83 & 8 & $74.52 \pm .07$ & 3.866 & $\mathbf{r}$ & 83 \\
\hline $\mathrm{FeS}_{2}$ & Pyrite & 120.082 & cub & 159.000 & 4 & $23.942 \pm .004$ & 5. 016 & $\mathbf{r}$ & $129,82,56$ \\
\hline $\mathrm{FeS}$ & Troilite & 87.916 & hex & 60.31 & 2 & $18.18 \pm .01$ & 4.835 & $r$ & 155 \\
\hline
\end{tabular}




\begin{tabular}{|c|c|c|c|c|c|c|c|c|c|}
\hline Formula & SULFIDES, & $\begin{array}{l}\text { TELLUR } \\
\text { Formula } \\
\text { Weight } \\
\text { grams }\end{array}$ & $\begin{array}{l}\text { DES, SE } \\
\text { Crystal } \\
\text { System }\end{array}$ & $\begin{array}{l}\text { ENIDES, } \\
\begin{array}{c}\text { Cell } \\
\text { Volume } \\
10^{-24} \mathrm{~cm}^{3}\end{array}\end{array}$ & $\begin{array}{l}\text { AND } \\
\text { Z }\end{array}$ & $\begin{array}{l}\text { SENIDES } \\
\text { Molar Volume } \\
\text { cm }^{3}\end{array}$ & $\begin{array}{c}\text { Density } \\
\text { grams } \mathrm{cm}^{-3}\end{array}$ & $\underset{{ }^{\circ} \mathrm{C}}{\text { Temp. }}$ & Ref. \\
\hline $\mathrm{FeS}_{2} *$ & Marcasite & 120.082 & orth & 81.622 & 2 & $24.58 \pm .02$ & 4.885 & 25 & 128,17 \\
\hline $\mathrm{FeSe}_{2}$ & Ferroselite & 213.77 & orth & 97.538 & 2 & $29.97 \pm .05$ & 7.22 & $\mathbf{r}$ & 62,9 \\
\hline $\mathrm{FeTe}_{2}$ & Frohbergite & 311.07 & orth & 127.62 & 2 & $38.44 \pm .07$ & 8. 092 & $\mathbf{r}$ & 60 \\
\hline $\mathrm{CoS}_{2}$ & Cattierite & 123.072 & cub & 169.57 & 4 & $25.53 \pm .04$ & 4.820 & $\mathbf{r}$ & 87 \\
\hline $\mathrm{CoSe}_{2}$ & Trogtalite & 216.86 & cub & 201.106 & 4 & $30.28 \pm .01$ & 7.162 & $r$ & 23 \\
\hline $\mathrm{NiS}_{2}$ & Vaesite & 122.842 & cub & 184.045 & 4 & $27.700 \pm .005$ & 4.435 & $\mathbf{r}$ & $10,79,84$ \\
\hline $\mathrm{NiSe}_{2}$ & & 216.63 & cub & 211.75 & 4 & $31.88 \pm .01$ & 6.795 & 20 & 61 \\
\hline $\mathrm{NiTe}_{2}$ & Melonite & 313.93 & hex & 68.81 & 1 & $41.4 \quad \pm .1$ & 7.58 & $\mathbf{r}$ & 151 \\
\hline $\mathrm{MnS}_{2}$ & Hauerite & 119.072 & cub & 227.18 & 4 & $34.202 \pm .006$ & 3.481 & 28 & 128 \\
\hline $\mathrm{PtAs}_{2} *$ & Sperrylite & 344.91 & cub & 212.56 & 4 & $32.00 \pm .05$ & 10.778 & $\mathbf{r}$ & 56,152 \\
\hline $\mathrm{RuS}_{2}$ & Laurite & 165.232 & cub & 175.6 & 4 & $26.4 \pm .2$ & 6.26 & $\mathbf{r}$ & $8,9,30,101$ \\
\hline $\mathrm{NiAs}_{2}$ & Rammelsbergite & 208.53 & orth & 97.675 & 2 & $29.42 \pm .04$ & 7.088 & 26 & $116,148,172$ \\
\hline $\mathrm{NiAs}_{2}$ & Pararammelsbergite & 208.53 & orth & 382.4 & 8 & $28.8 \pm .1$ & 7.24 & $\mathbf{r}$ & 78,105 \\
\hline $\mathrm{FeAs}_{2}$ & Loellingite & 205.67 & orth & 91.36 & 2 & $27.51 \pm .02$ & 7.476 & 26 & 116,148 \\
\hline $\begin{array}{l}\mathrm{CoAs}_{2} \\
(\mathrm{Co}, \mathrm{Fe}) \mathrm{As}_{2}\end{array}$ & $\begin{array}{l}\text { Co-safflorite } \\
\text { Safflorite }\end{array}$ & 208.76 & mon & 92.706 & 2 & $27.92 \pm .02$ & 7.477 & 26 & 116,148 \\
\hline $\mathrm{Co}_{0.5} \mathrm{Fe}_{0.5} \mathrm{As}_{2}$ & & 207.72 & orth & 92.23 & 2 & $27.78 \pm .06$ & 7.477 & 26 & 116,148 \\
\hline $\mathrm{MoS}_{2}$ & Molybdenite & 160.082 & hex & 106.35 & 2 & $32.03 \pm .01$ & 4.998 & 26 & 143 \\
\hline $\mathrm{WS}_{2}$ & Tungstenite & 247.992 & hex & 106.50 & 2 & $32.07 \pm .02$ & 7.733 & 26 & 146 \\
\hline FeAsS * & Arsenopyrite & 162.826 & $\operatorname{tri}$ & 175.49 & 4 & $26.42 \pm .05$ & 6.163 & $\mathbf{r}$ & 93 \\
\hline $\mathrm{FeSbS} *$ & Gudmundite & 209.676 & mon & 399.1 & 8 & $30.05 \pm .1$ & 6.978 & $\mathbf{r}$ & 18 \\
\hline$(\mathrm{Co}, \mathrm{Fe}) \mathrm{AsS}$ * & Glaucodot & 164.87 & orth & 1063. & 24 & 26.7 & 6.17 & $r$ & 9 \\
\hline
\end{tabular}


SULFIDES, TELLURIDES, SELENIDES, AND ARSENIDES

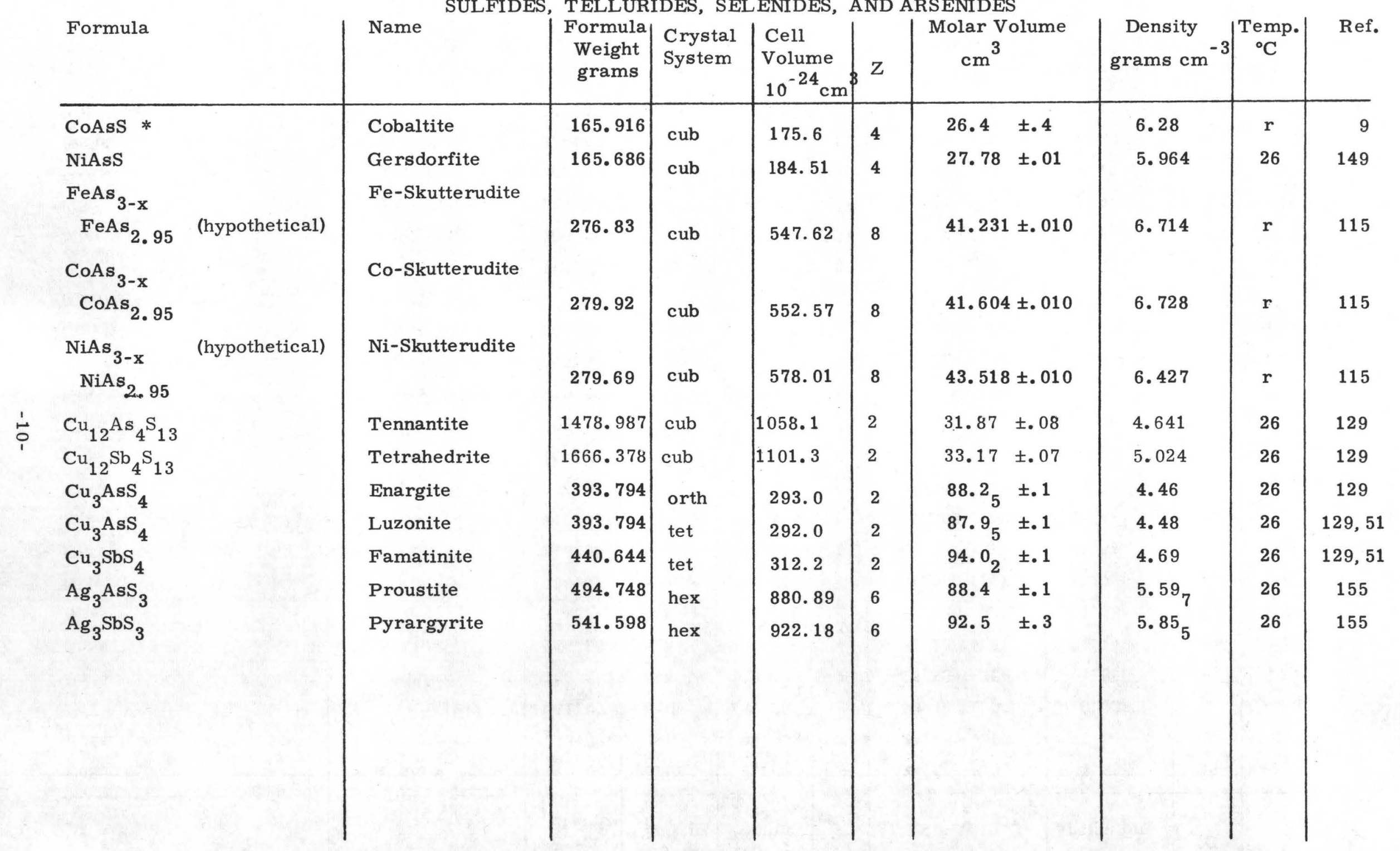




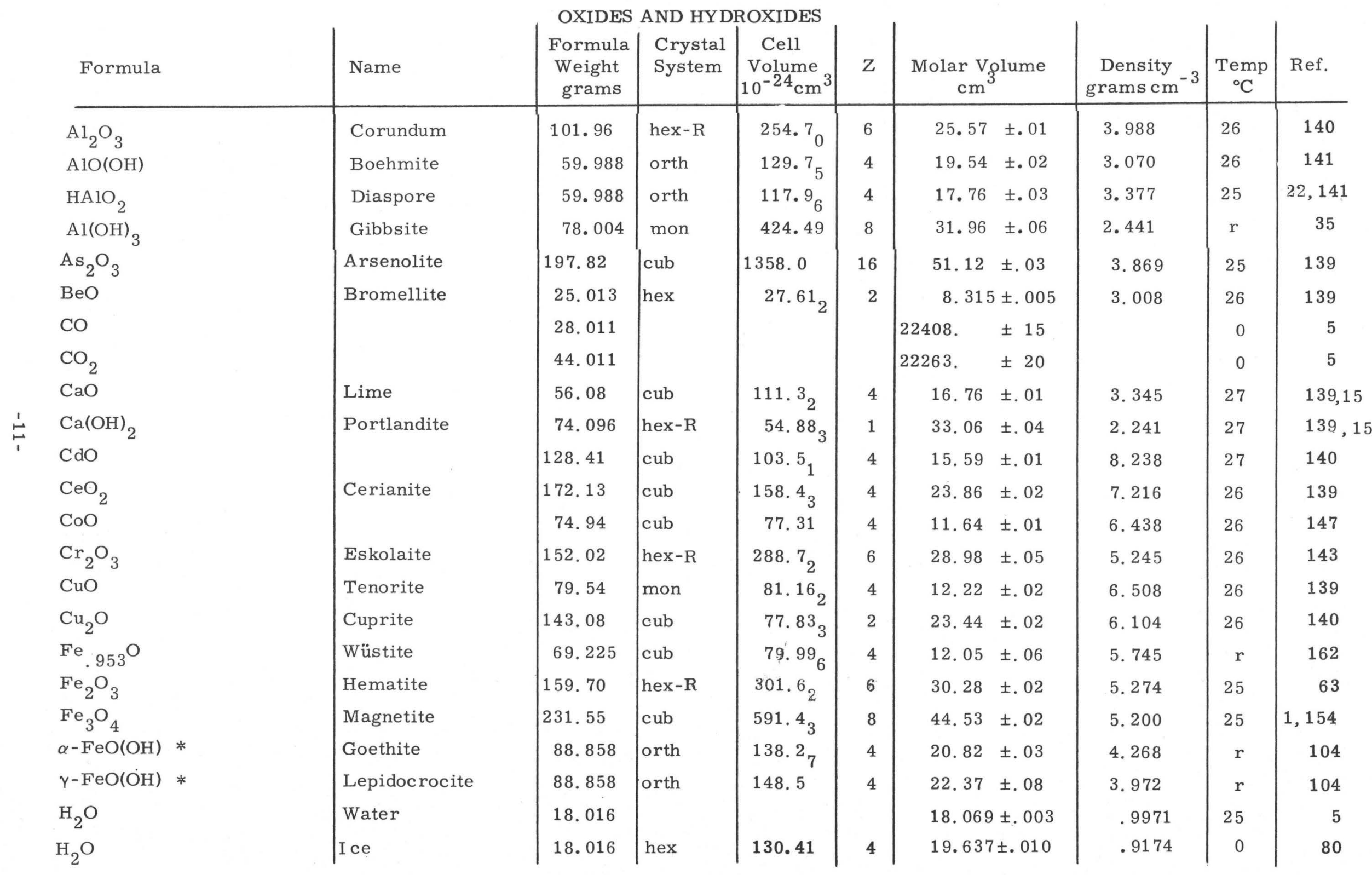




\begin{tabular}{|c|c|c|c|c|c|c|c|c|c|}
\hline Formula & Name & $\begin{array}{c}\text { OXIDES } \\
\text { Formula } \\
\text { Weight } \\
\text { grams }\end{array}$ & $\begin{array}{l}\text { AND HYD } \\
\text { Crystal } \\
\text { System }\end{array}$ & $\left\{\begin{array}{c}\text { Ce } 11 \\
\text { Volume } \\
10^{-24} \mathrm{~cm}^{3}\end{array}\right.$ & $\mathrm{Z}$ & $\begin{array}{c}\text { Molar Volume } \\
\mathrm{cm}^{3}\end{array}$ & $\begin{array}{c}\text { Density } \\
\text { grams cm }\end{array}$ & $\begin{array}{c}\text { Temp } \\
{ }^{\circ} \mathrm{C}\end{array}$ & Ref. \\
\hline $\mathrm{HfO}_{2}$ & Hafnia & 210.50 & mon & 138.30 & 4 & $20.82 \pm .01$ & 10.108 & $\mathrm{r}$ & 2 \\
\hline $\mathrm{HgO}$ & Montroydite & 216.61 & orth & $128.3_{1}$ & 4 & $19.32 \pm .02$ & 11.211 & 25 & 147 \\
\hline MgO & Periclase & 40.32 & cub & $74.70_{9}$ & 4 & $11.25 \pm .01$ & 3.584 & 25 & 126,139 \\
\hline $\mathrm{Mg}(\mathrm{OH})_{2}$ & Brucite & 58.336 & hex-R & $40.90_{3}$ & 1 & $24.64 \pm .03$ & 2.368 & 26 & 144 \\
\hline $\mathrm{MnO}$ & Manganosite & 70.94 & cub & $87.81_{2}$ & 4 & $13.22 \pm .01$ & 5.365 & 26 & 143,53 \\
\hline $\mathrm{MnO}_{2}$ & Pyrolusite & 86.94 & tet & 55.16 & 2 & $16.61 \pm .06$ & 5.233 & $\mathrm{r}$ & 138 \\
\hline $\mathrm{Mn}_{2} \mathrm{O}_{3}$ & Bixbyite & 157.88 & cub & $833.5_{0}$ & 16 & $31.38 \pm .03$ & 5.032 & 25 & 147 \\
\hline $\mathrm{Mn}_{3} \mathrm{O}_{4}$ & Hausmanite & 228.82 & tet & 623.6 & 4 & $46.96 \pm .08$ & 4.873 & $r$ & 158 \\
\hline $\mathrm{MoO}_{3}$ & Molybdite & 143.95 & orth & $202.9_{8}$ & 4 & $30.72 \pm .02$ & 4.686 & 26 & 141 \\
\hline $\mathrm{NiO}$ & Bunsenite & 74. 71 & cub & 72.877 & 4 & $10.97 \pm .01$ & 6.808 & 26 & 139 \\
\hline $\mathrm{PbO}$ & Litharge & 223.21 & tet & $79.40_{2}$ & 2 & $23.91 \pm .02$ & 9.334 & 27 & 140 \\
\hline $\mathrm{PbO}$ & Massicot & 223.21 & orth & $153.7_{6}$ & 4 & $23.15 \pm .02$ & 9.641 & 27 & 140 \\
\hline $\mathrm{SO}_{2}$ & & 64.066 & & & & $21894 . \quad \pm 15$ & & 0 & 5 \\
\hline $\mathrm{Sb}_{2} \mathrm{O}_{3}$ & Senarmontite & 291.52 & cub & 1386.9 & 16 & $52.21 \pm .03$ & 5.583 & 26 & 141 \\
\hline $\mathrm{Sb}_{2} \mathrm{O}_{3}$ & Valentinite & 291.52 & orth & $332.1_{3}$ & 4 & $50.01 \pm .06$ & 5.829 & 25 & 148 \\
\hline $\mathrm{SiO}_{2} *$ & $\alpha$-Quartz & 60.09 & hex-R & $113.01_{3}$ & 3 & $22.690 \pm .005$ & 2.648 & 25 & 46 \\
\hline $\mathrm{SiO}_{2}$ & $\alpha$-Cristobalite & 60.09 & tet & $170.9_{5}$ & 4 & $25.74 \quad \pm .02$ & 2.334 & 25 & 148 \\
\hline $\mathrm{SiO}_{2}$ & $\beta$-Cristobalite & 60.09 & cub & $363.7_{2}$ & 8 & $27.38 \pm .02$ & 2.194 & 405 & 134 \\
\hline $\mathrm{SiO}_{2}$ & $\alpha$-Tridymite & 60.09 & hex & & & $26.53 \pm .20$ & 2.265 & $r$ & 43 \\
\hline $\mathrm{SiO}_{2}$ & $\beta$-Tridymite & 60.09 & hex & $182.0_{8}$ & 4 & $27.42 \pm .03$ & 2.192 & 405 & 134 \\
\hline $\mathrm{SiO}_{2} *$ & Coesite & 60.09 & mon & $548.3_{7}$ & 16 & $20.64 \pm .05$ & 2.911 & 25 & 128 \\
\hline $\mathrm{SiO}_{2} *$ & Stishovite & 60.09 & tet & 46.541 & 2 & $14.016 \pm .01$ & 4.287 & $\mathrm{r}$ & 24 \\
\hline $\mathrm{SiO}_{2}$ & $\beta$-Quartz & 60.09 & hex-R & 118.15 & 3 & $23.72 \pm .01$ & 2.533 & 575 & 174 \\
\hline $\mathrm{SiO}_{2}$ & Keatite & 60.09 & tet & 479.2 & 12 & $24.05 \pm .30$ & 2.498 & $r$ & 124 \\
\hline
\end{tabular}




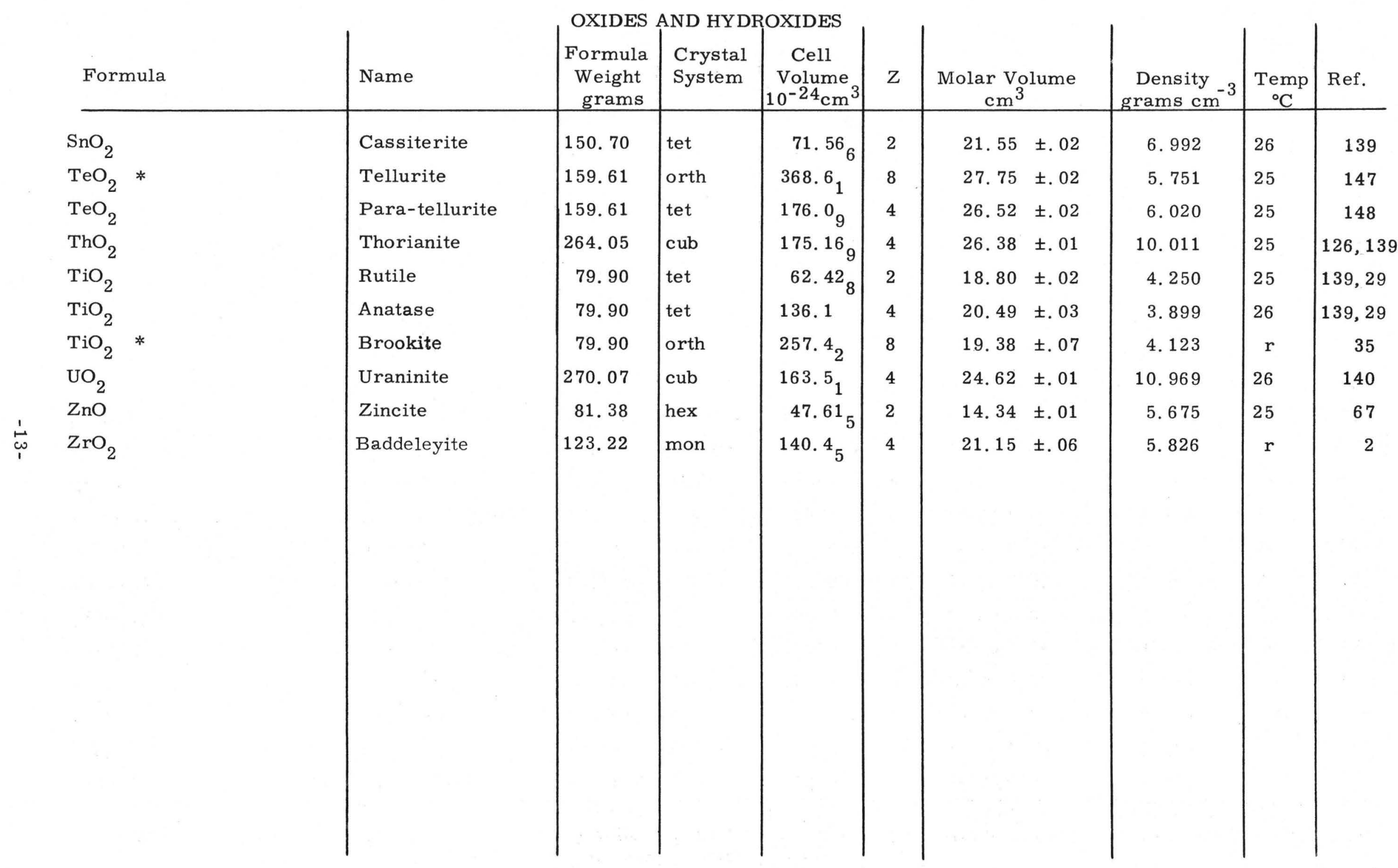




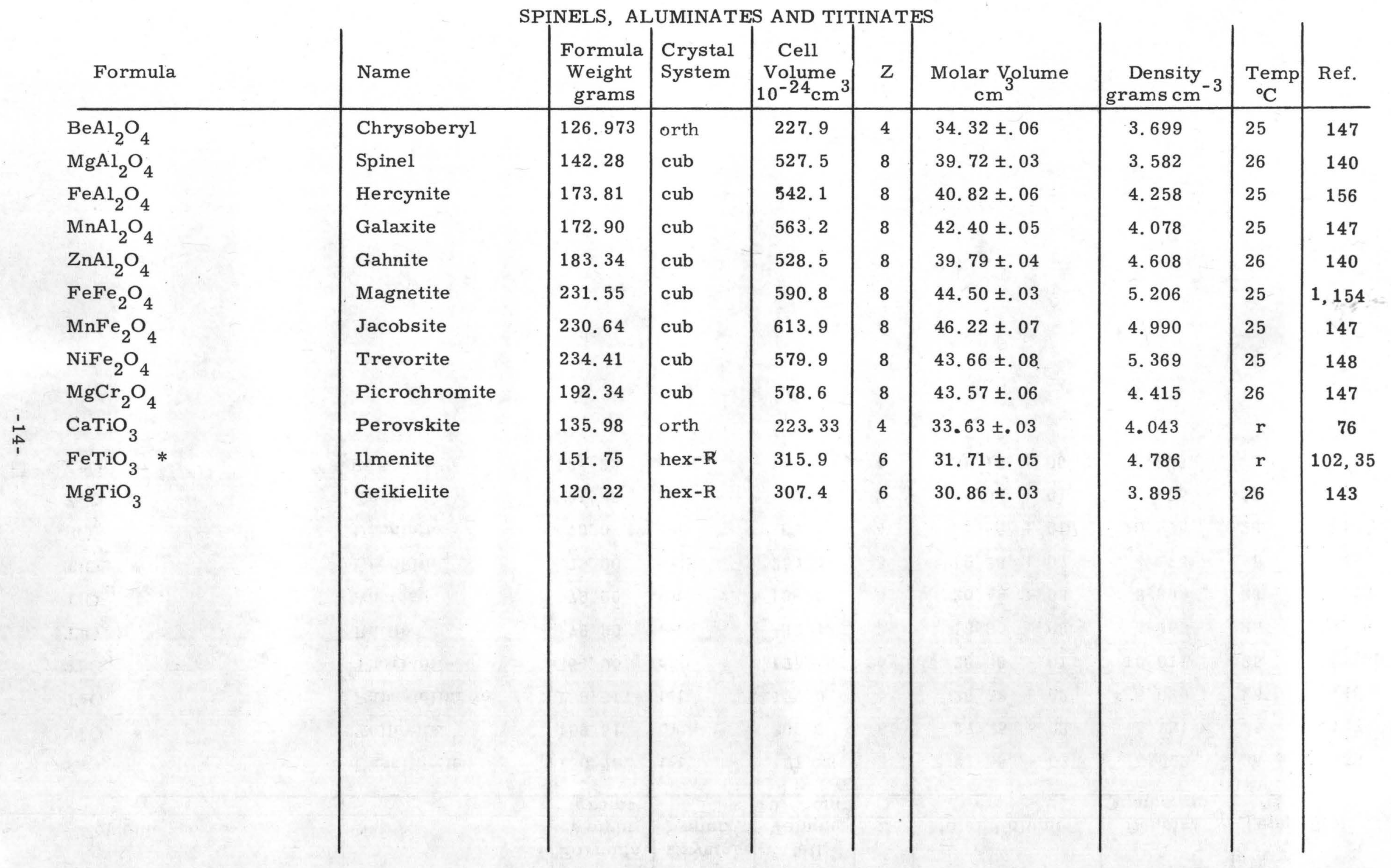




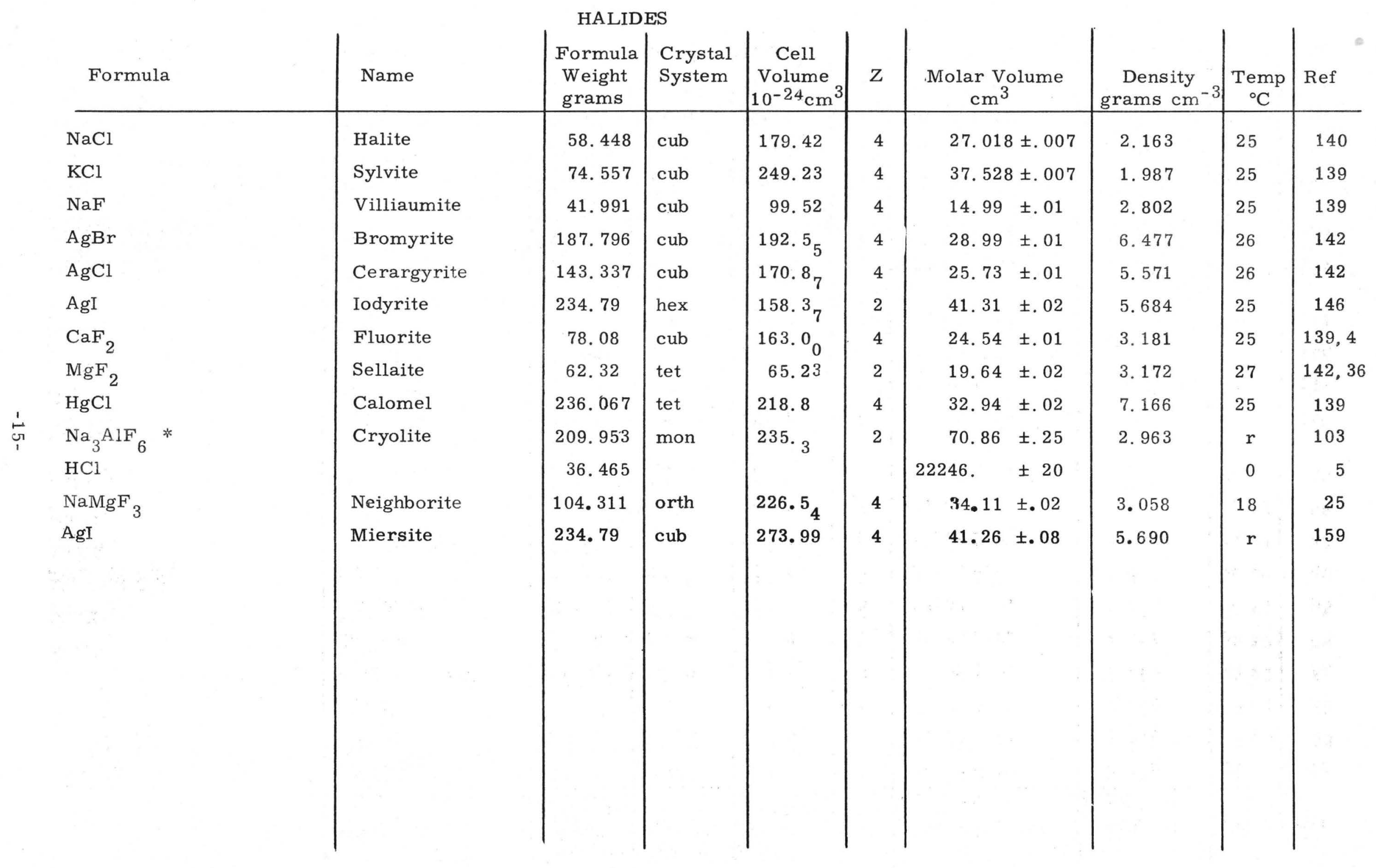




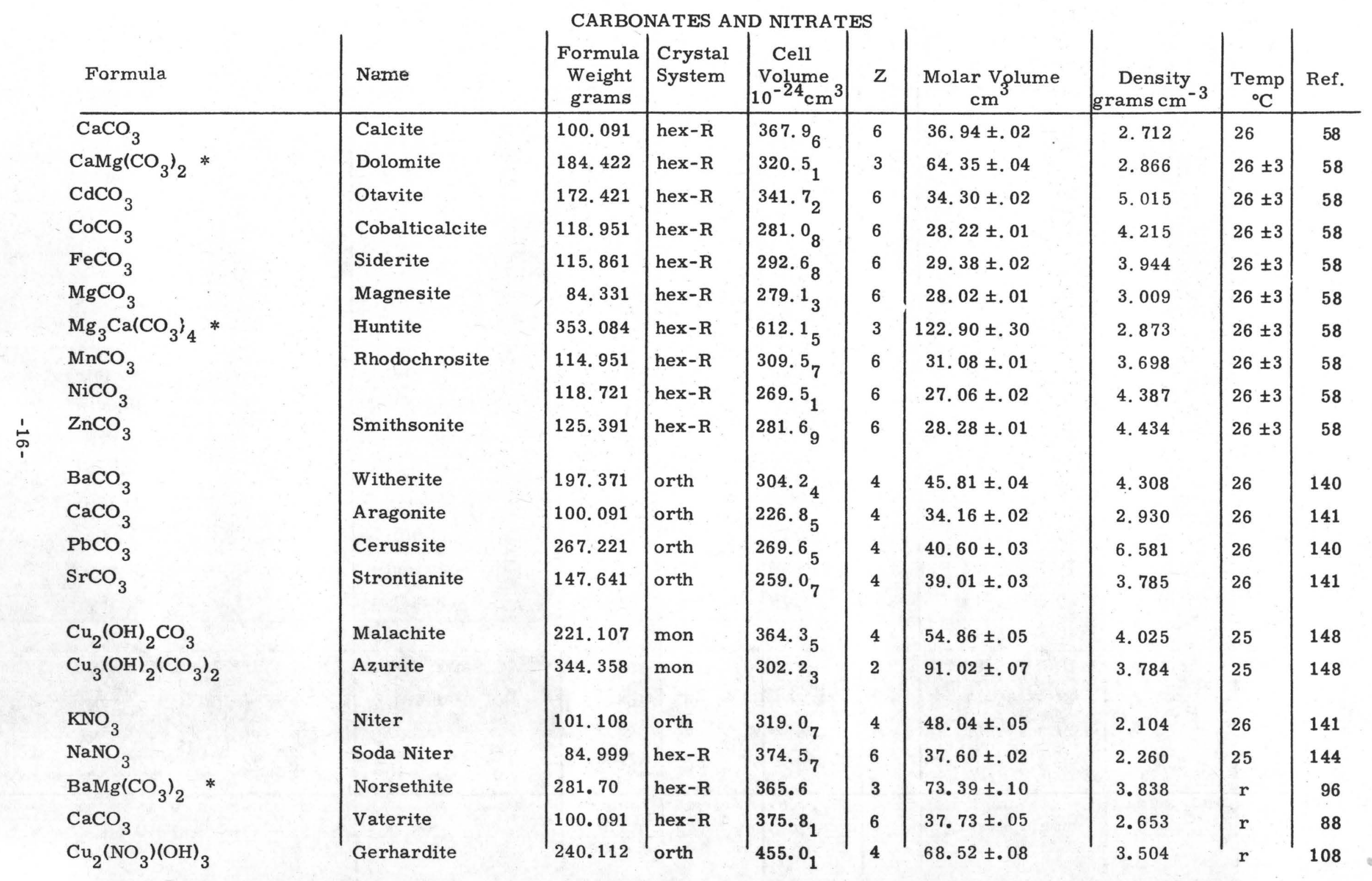




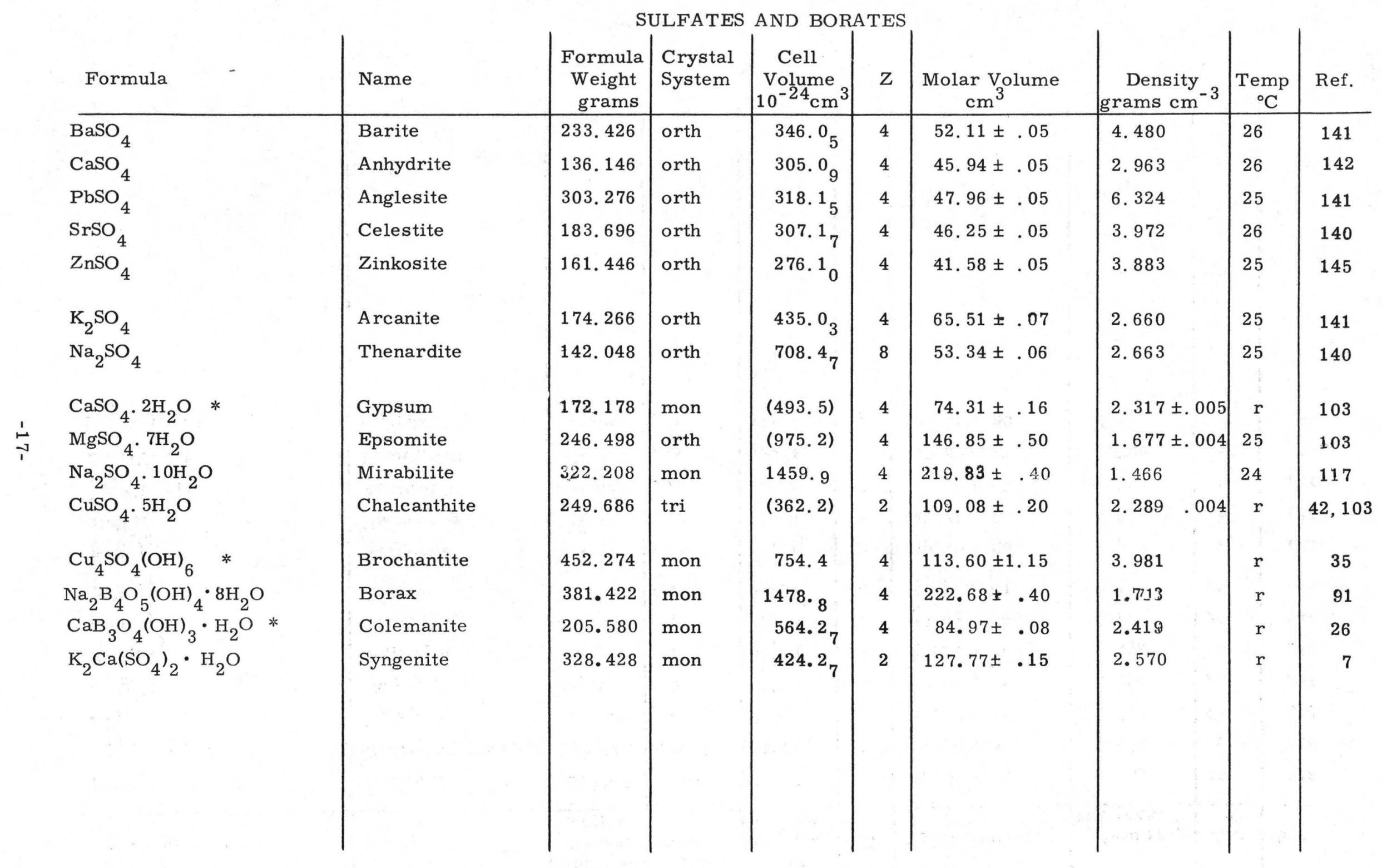




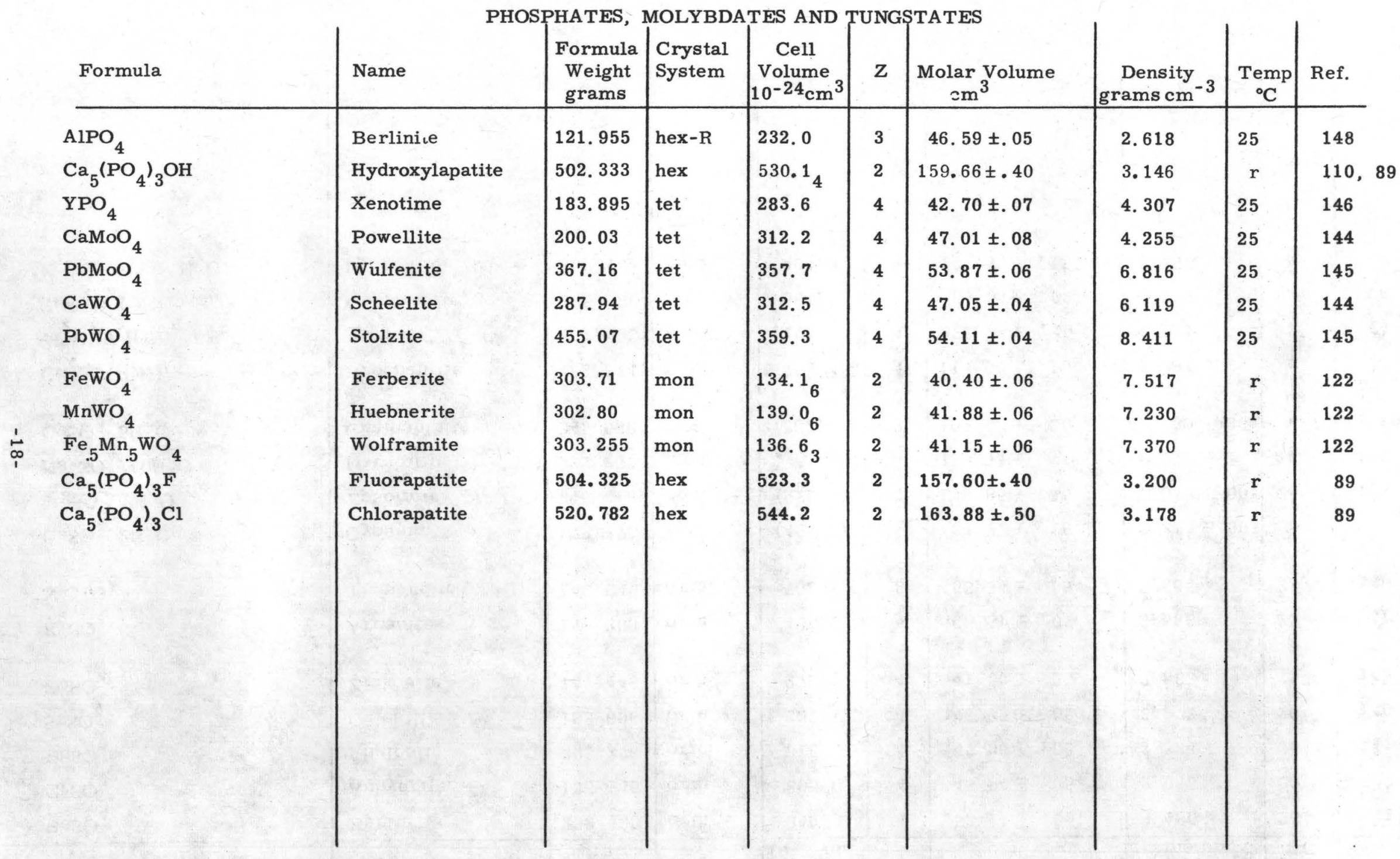




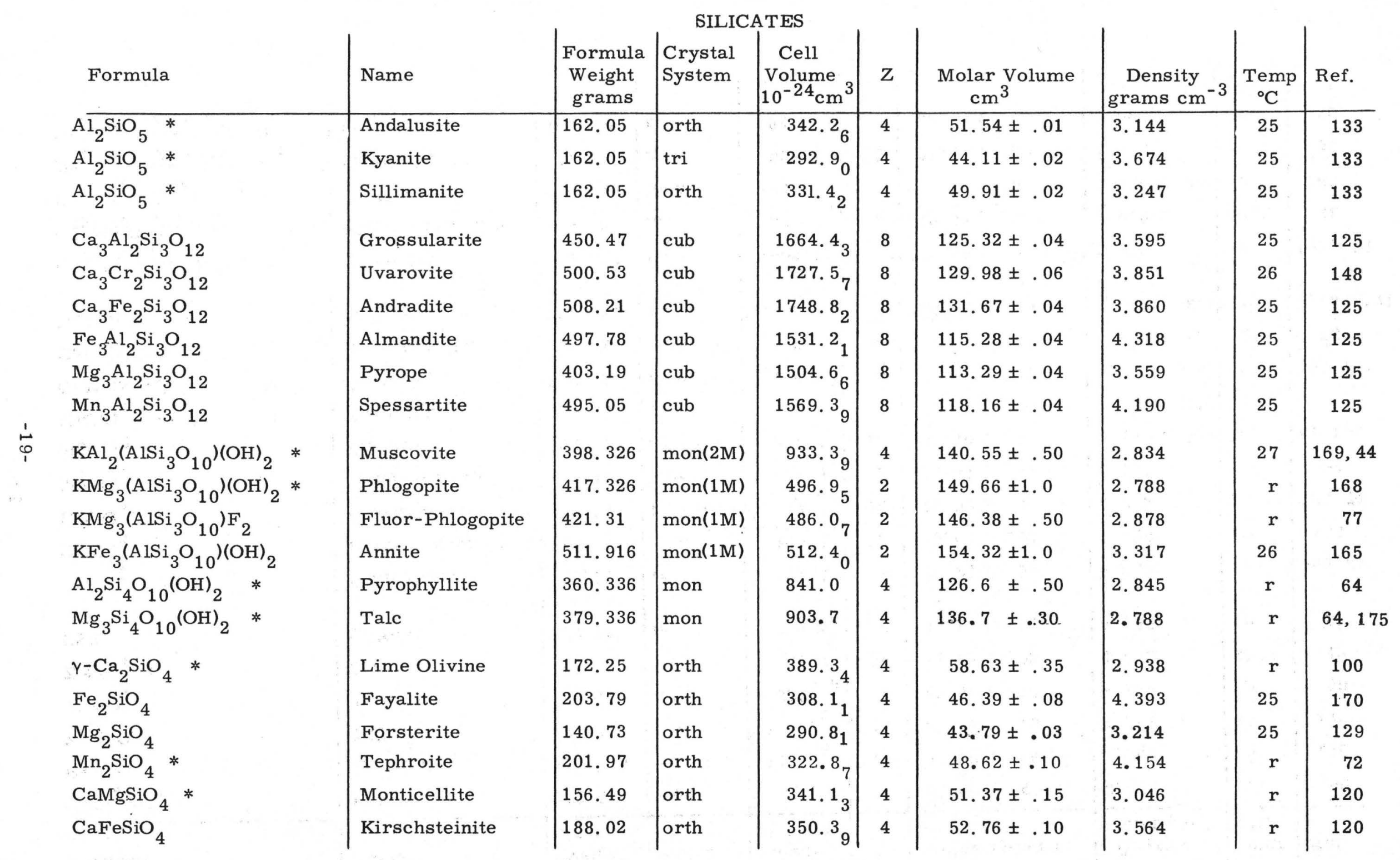




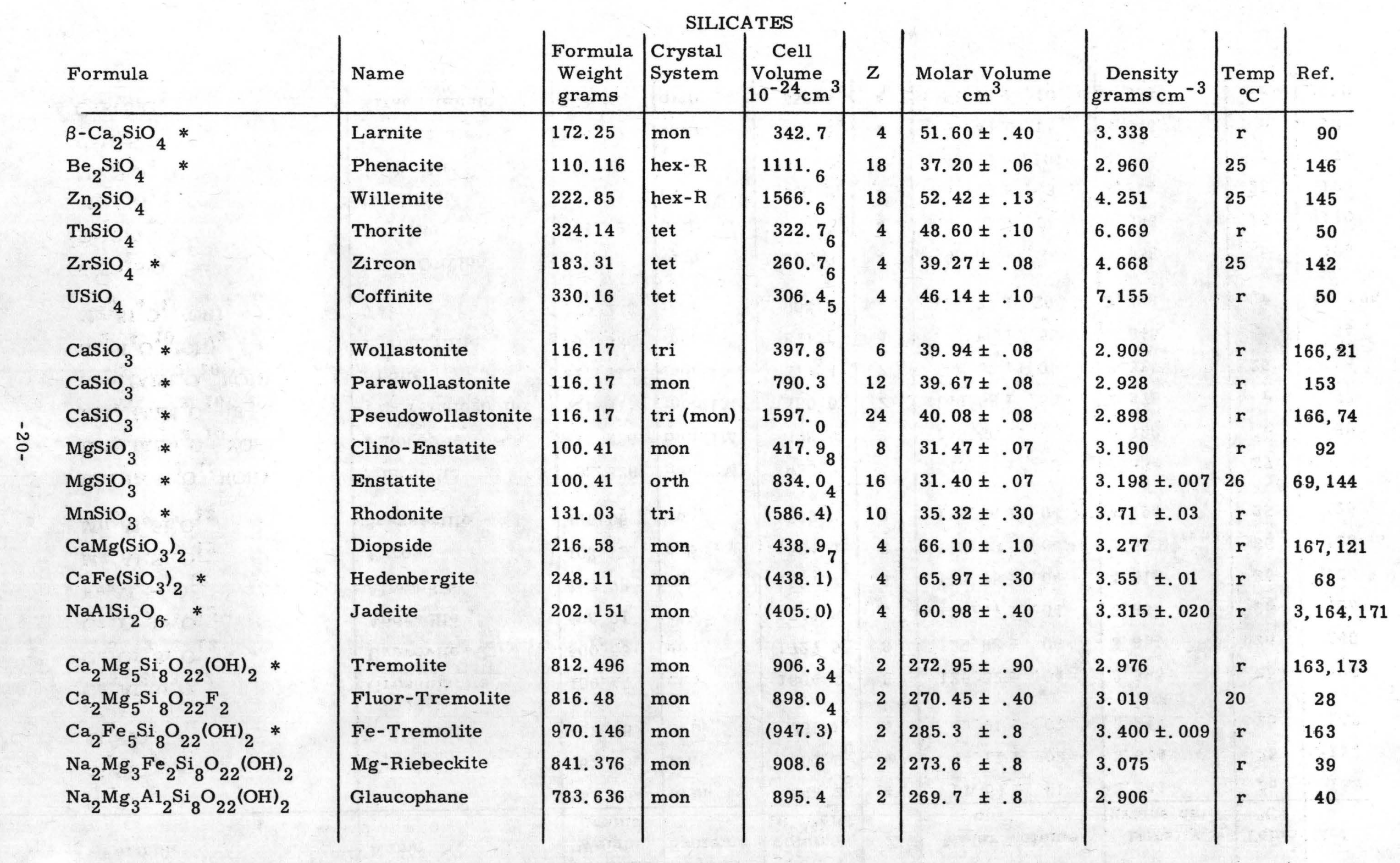




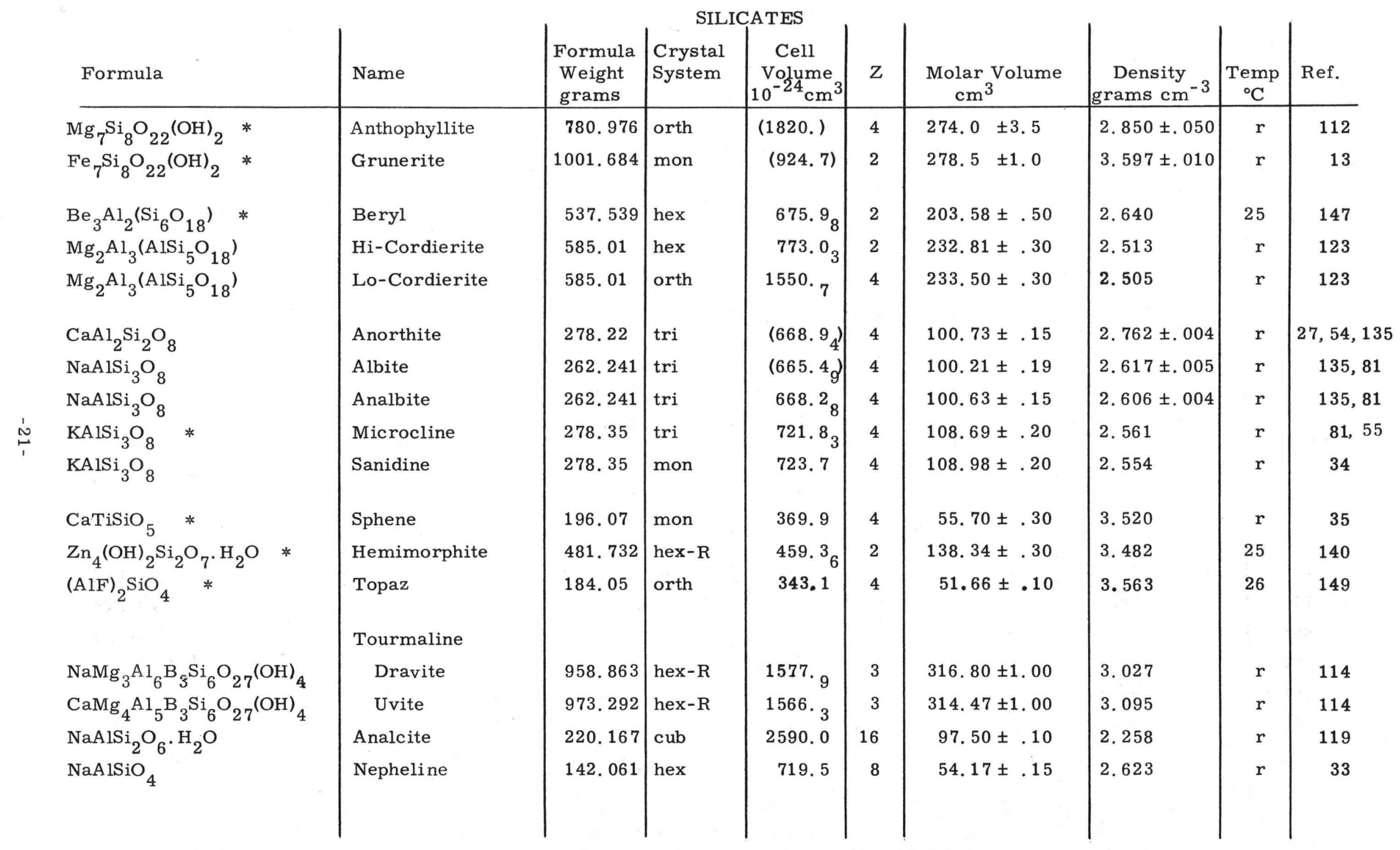




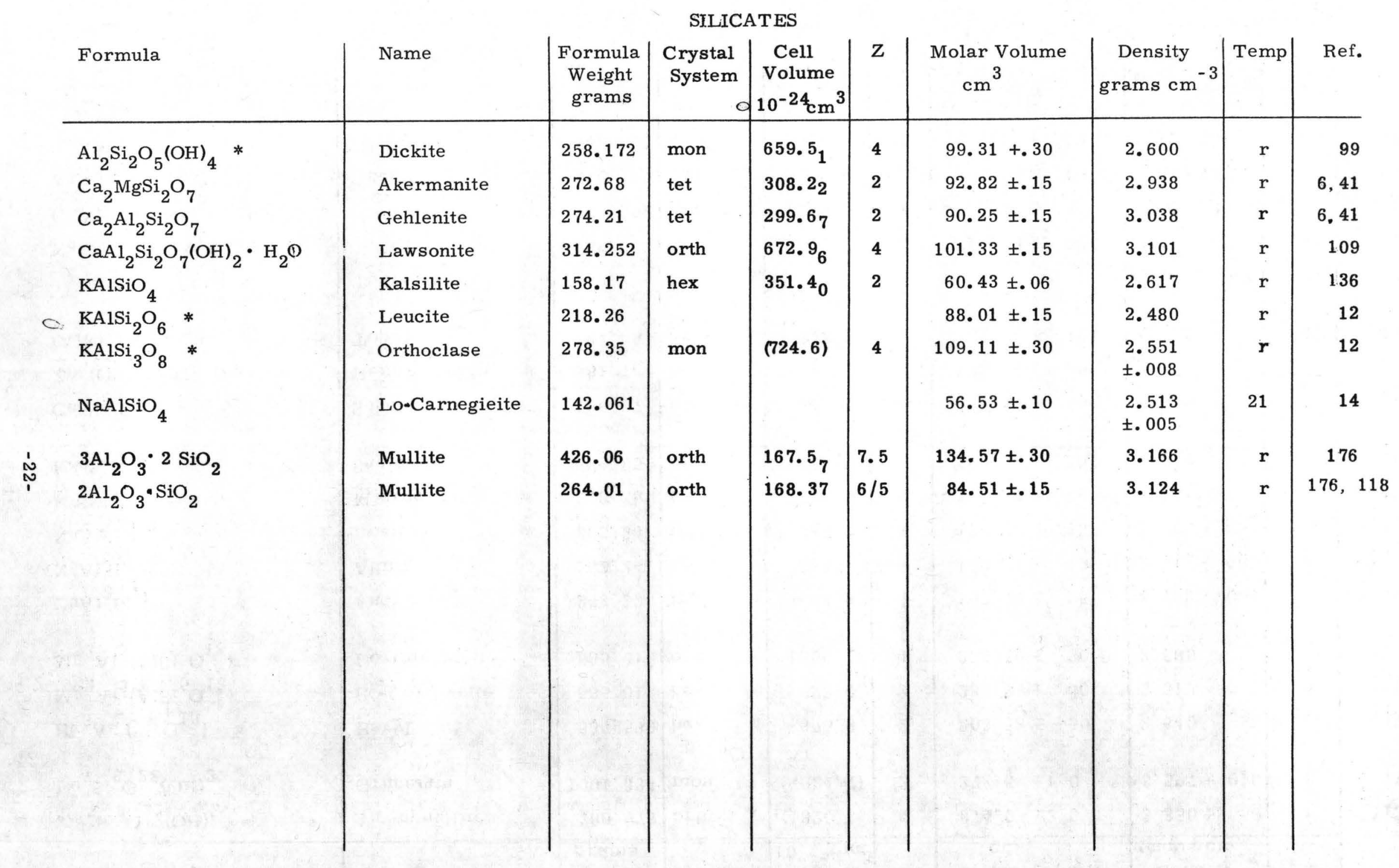


1. Abrahams and Calhoun, Acta Cryst. $6,105,1953$.

2. Adam and Rodgers, Acta Cryst. 12, 951, 1959.

3. Adams and Gibson, Pro. Nat. Acd. Sci. 15, 713, 1929.

4. Allen, Am. Min. 37, 910, 1952.

5. American Institute of Physics Handbook, McGraw Hill, New York, 1957.

6. Andrews, Min. Mag. 28, 374, 1948.

7. Aruja, Min. Mag. 31, 943, 1958.

8. Bannister, Min. Mag. 23, 195, 1932.

9. Berry and Thompson, Mem. 85, Geol. Soc. Am., 281 p., 1962.

10. Bethke, P. M., unpublished data.

11. Bethke and Barton, U. S. Geol. Surv. Prof. Paper 424B, 266, 1961.

12. Birch and others, Geol. Soc. Am., Special Paper 36, 1942.

13. Bowen and Schairer, Am. Min. 543, 1935.

14. Bowen, Am. J. Sci., XLIII, 115, 1917.

15. Brunauer, Kantro and Weise, Can. J. Chem. 34, 729, 1956.

16. Buerger, Am. Min. 20, 36, 1935.

17. — Zeit. Krist. 97, 504, 1937.

18. - Am. Min. 23, 4, 1939.

19. —, Am. Min. 27, 301, 1942.

20. —, Am. Min. 32, 415, 1947.

21. - Pro. Nat. Acd. Sci. 42, 113, 1956.

22. Busing and Levey, Acta Cryst. 11, 798, 1958.

23. B $\phi$ hm and others, Acta Chem. Scand. 9, 1510, 1955.

24. Chao, Fahey, Littler, and Milton, J. Geophys. Res. 67, 419, 1962.

25. Chao and others, Amer. Min. 46, 379, 1961. 
26. Christ, Am. Min. $\underline{41}, 569,1956$.

27. Cole, Sorum and Taylor, Acta. Cryst. 4, 20, 1951.

28. Comeforo and Kohn, Am. Min. 39, 537, 1954.

29. Cromer and Herrington, J. Am. Chem. Soc. 77, 4708, 1955.

30. deJong and Hoog, Rec. Trav. Chim. $\underline{46}, 173,1927$.

31. Djurle, Acta Chem. Scand. 12, 1415, 1958.

32. - Acta Chem. Scand. 12, 1427, 1958.

33. Donnay, Ann. Rept. Dir. Geophys. Lab. 56, 239, 1957.

34. Donnay and Donnay, Am. J. Sci., Bowen Vol., 115, 1952.

35. Donnay and Nowacki, Crystal Data, Geol. Soc. Am., Memoir 60 , 1954.

36. Duncanson and Stevenson, Pro. Phy. Soc. 72, 1001, 1958.

37. Earley, Am. Min. 34, 433, 1949.

38. - Am. Min. 35, 337, 1950.

39. Ernst, Geochim et Cosmochim. Acta. 19, 10, 1960.

40. —, Am. J, Sci. 259, 735, 1961.

41. Ferguson and Buddington, Am. J. Sci. 50, 133, 1920.

42. Fisher, Am. Min. 37, 95, 1952.

43. Fleming and Lynton, Phy. Chem. Glasses 1, 148, 1960.

44. Fournier, R. O., Private communication, June 1960.

45. Francombe, Brit. J. Appl. Phys. $\underline{9}, 415,1958$.

46. Frondel and Hurlbut, J. Chem. Phys. 23, 1215, 1955.

47. Frueh, Geol. Soc. Am. Bull. 67, 1697, 1956.

48. —, Am. Min. 44, 693, 1959.

49. — Zeit. Krist. 112, 44, 1959.

50. Fuchs and Gebert, Amer. Min. 43, 243, 1958.

51. Gaines, Am. Min. $\underline{42}, 766,1957$. 
52. Goldschmidt, Norsk. Videns, Akad. Oslo I Maf. Nat. Klasse, no. 8, 1927.

53. Goldsmith and Graf, Geochim. et Cosmochim. Acta 11, 310,1957.

54. Goldsmith and Laves, Zeit. Krist. 106, 213, 1955.

55. - Geochim. et Cosmochim. Acta. 5, 1, 1954.

56. Gordon, Am. Min. 36, 918, 1951.

57. Goryunova and Fedorova, Sov. Phys. Solid State, 1, 307, 1959.

58. Graf, Am. Min. 46, 1283, 1961.

59. Grфnvold, Haraldsen and Kjekshus, Acta. Chem. Scand. 14, $1879,1960$.

6c. Grфnvold, Haraldsen and Vihovde, Acta Chem. Scand. 8, 1927, 1954.

61. Grфnvold, and Jacobsen, Acta Chem. Scand. 10, 1440, 1956.

62. Grфnvold and Westrum, Inorg. Chem. 1, 36, 1962.

63. - J. Am. Chem. Soc. 81, 1780, 1959.

64. Gruner, Zeit. Krist. 88, 412, 1934.

65. Güntert and Faessler, Zeit. Krist. 107, 357, 1956.

66. Hall, U. S. Geol. Surv. Prof. Paper 424B, 271, 1961.

67. Heller, McGannon, and Weber, J. Appl. Phys. 21, 1283, 1950.

68. Hess, Am. Min. 34, 621, 1949.

69. — - Am. J. Sci., Bowen Vol., 173, 1952.

70. Hewitt, Econ. Geol. 43, 408, 1948.

71. Hiller, Neues Jahrb. Mineral Monatsch, 265, 1951.

72. Hurlbut, Am. Min. 46, 549, 1961.

73. Ievins, Straumanis, and Karlsons, Zeit. Phys. Chem. 40B, $347,1938$.

74. Jeffery and Heller, Acta Cryst. 6, 807, 1953.

75. Jurriaanse, Zeit. Krist. 90, 322, 1935.

76. Kay and Bailey, Acta Cryst. 10, 219, 1957. 
77. Kohn and Hatch, Ám. Min. 40, 10, 1955.

78. Kullerud, G., Unpublished data.

79. Kullerud and Yund, J. Petrology 3 , 126, 1962.

80. LaPlaca and Post, Acta Cryst. 12, 951, 1959.

81. Laves, J. Geol. 60, 549, 1952.

82. Lepp, Am. Min. 41, 347, 1956.

83. Lundquist, Ark. Kem. Min. Geol. 17B, n. 12, 1943.

84. - Ark. Kem. Min. Geol. 24A, n. 21, 1947.

85. - Ark. Kem. Min. Geol. 24A, n. 22, 1947.

86. - Ark. Kem. Min. Geol. 24A, n. 23, 1947.

87. Lundquist and Westgren, Zeit. anorg. Chem. 239, 85, 1938.

88. McConnell, Min. Mag. 32, 534, 1960.

89. McConnell, Science 136, 241, 1962.

90. Midgley, Acta Cryst. 5, 307, 1952.

91. Morimoto, Min. J. (Japan) 2, 1, 1956.

92. Morimoto, Ann. Rept. Dir. Geophys. Lab. 58, 193, 1959.

93. Morimoto and Clark, Am. Min. 46, 1448, 1961.

94. Morimoto and Kullerud, Am. Min. $\underline{46}, 1270,1961$.

95. Mosburg and others, U. S. Geol. Surv. Prof. Paper 424C, 347, 1961.

96. Mrose and others, Am. Min. 46, 420, 1961.

97. Murdoch and Berry, Am. Min. 39, 475, 1954.

98. Nelson and Riley, Pro. Phy. Soc. 57, 477, 1945.

99. Newman, Min. Mag. 32, 683, 1961.

100. O'Daniel and Tscheischwili, Zeit. Krist. 104, 124, 1942.

101. Oftedal, Zeit. Phys. Chem. 135, 291, 1928.

102. Palache, Berman, and Frondel, Dana's System of Mineralogy, 7th edition, vol. 1, John Wiley, New York, 1944. 
103. Palache, Berman, and Frondel, Dana's System of Mineralogy, 7th edition, vol. 2, John Wiley, New York, 1951.

104. Peacock, Trans. Roy. Soc. Canada (IV) 36, 107, 1942.

105. - Univ. Toronto Stud. Geol. Ser. 42, 101, 1939.

106. - Am. Min. 27, 229, 1942.

107. Peacock and McAndrew, Am. Min. 35, 425, 1950.

108. Pistorious, Zeit. Krist. 113, 478, 1960.

109. - Am. Min. 46, 982, 1961.

110. Posner, Perloff, and Diorio, Acta Cryst. 11, 308, 1958.

111. Primak, Kaufman, and Ward, J. Am. Chem. Soc. 70, 2043, 1948.

112. Rabbitt, Am. Min. 33, 263, 1949.

113. Rahlfs, Zeit. Phys. Chem. B31, 157, 1936.

114. Robbins, Ann. Rept. Dir. Geophys. Lab. 58, 137, 1959.

115. Roseboom, Am. Min. 47, 310, 1962.

116. Unpublished data.

117. Ruben and others, J. Am. Chem. Soc. 83, 821, 1961.

118. Sadanga, Tokonami, and Takeuchi, Acta Cryst. 15, 65, 1962.

119. Saha, Am. Min. 44, 300, 1959.

120. Sahama and Hytonen, Am. Min. $\underline{43}, 862,1958$.

121. Sakata, Jap. J. Geol. Geog. 28, 161, 1957.

122. Sasaki, Min. J. 2, 375, 1959.

123. Schreyer and Schairer, J. Petrology 2, 324, 1961.

124. Shropshire, Keat, and Vaughan, Zeit. Krist. 112, 409, 1959.

125. Skinner, Am. Min. 41, 428, 1956.

126. - Am. Min. $\underline{42}, 39,1957$.

127. — Am. Min. $\underline{46}, 1399,1961$. 
128. Skinner, B. J., Written Communication, June 1962.

129. Unpublished data.

130. Skinner and Barton, Am. Min. 45, 612, 1960.

131. Skinner, Barton, and Kullerud, Econ. Geol. 54, 1040, 1959.

132. Skinner and Bethke, Am. Min. $\underline{46}, 1382,1961$.

133. Skinner, Clark, and Appleman, Am. J. Sci. 259, 651, 1961.

134. Smith, Ann. Rept. Dir. Geophys. Lab. 52, 61, 1953.

135. Smith, Min. Mag. 31, 47, 1956.

136. Smith and Tuttle, Am. J. Sci. 255, 282, 1957.

137. Straumanis, J. Appl. Phys. 20, 726, 1949.

138. Structure Reports, 184, 1951.

139. Swanson and Tatge, U. S. Nat. Bur. Stds. Circular 539, v. 1, Washington, D. C., 1953.

140. Swanson and Fuyat, U. S. Nat. Bur. Stds. Circular 539, v. 2, Washington, D. C., 1953.

141. Swanson, Fuyat, and Ugrinic, U. S. Nat. Bur. Stds. Circular 539 , v. 3, Washington, D. C., 1954.

142. - U. S. Nat. Bur. Stds. Circular 539, v. 4 , Washington, D. C., 1955.

143. Swanson, Gilfrich, and Ugrinic, U. S. Nat. Bur. Stds. Circular 539, v. 5, Washington, D. C., 1955.

144. Swanson, Gilfrich, and Cook, U. S. Nat. Bur. Stds. Circular 539 , v. 6, Washington, D. C., 1956.

145. - U. S. Nat. Bur. Stds. Circular 539, v. 7, Washington, D. C., 1957.

146. Swanson and others, U. S. Nat. Bur. Stds. Circular 539, v. 8, Washington, D. C., 1959.

147. - U. S. Nat. Bur. Stds. Circular 539, v. 9 , Washington, D. C., 1960.

148. - U. S. Nat. Bur. Stds. Circular 539, v. 10 , Washington, D. C., 1960. 
149. - U. S. Nat. Bur. Stds. Monogr. 25, sec. 1, Washington, D. C, 1962 .

150. Taylor and Underwood, Acta Cryst. 13, 361, 1960.

151. Tengner, Zeit. anorg. Chem. 239, 126, 1938.

152. Thomassen, Zeit. Phys. Chem. $\underline{4}$, Abt. B, 277, 1929.

153. Tolliday, Nature $\underline{182}, 1012,1958$.

154. Tombs and Rooksby, Acta Cryst. $\underline{4}, 474,1951$.

155. Toulmin, P. J., in press.

156. Turnock, A., private communication, February 1961.

157. Ulrich and Zachariasen, Zeit. Krist. 62, 260, 1925.

158. Van Hook and Keith, Am. Min. 43, 69, 1958.

159. Waldbaum, D. R., private communication, August 1961.

160. Wasserstein, Am. Min. 36, 102, 1951.

161. Wiese and Muldawer, Phys. and Chem. Solids 15, 13, 1960.

162. Willis and Rooksby, Acta Cryst. $\underline{6}, 827,1953$.

163. Winchell, Am. Min. 30, 33, 1945.

164. Wolfe, Am. Min. 40, 249, 1955.

165. Wones, D., private communication, February 1961.

166. Wright, Am. J. Sci. 189, 6, 1915.

167. Yoder, J. Geol. 60, 364, 1952.

168. Yoder and Eugster, Geochim. et Cosmochim. Acta $\underline{6}, 157,1954$.

169. - Geochim. et Cosmochim. Acta $\underline{8}, 225,1955$.

170. Yoder and Sahama, Am. Min. 42, 475, 1957.

171. Yoder and Weir, Am. J. Sci. 249, 683, 1951.

172. Yund, Ann. Rept. Dir. Geophys. Lab. Wash. 58, 148, 1959.

173. Zussnan, Acta Cryst. 12, 309, 1959. 
174. Calculated from $\alpha$-quartz cell and thermal expansion of Jay, Pro. Roy. Soc., A142, 237, 1933.

175. Stemple and Brindley, J. Am. Ceram. Soc. $\underline{43}, 34,1960$.

176. Agrell and Smith, J. Am. Ceram. Soc. 43, 69, 1960. 